\title{
Evaluation of a Product Containing Xyloglucan and Pea Protein on Skin Barrier Permeability
}

\author{
Michela Campolo Marika Lanza Alessia Filippone Irene Paterniti \\ Giovanna Casili Sarah A. Scuderi Alessio Ardizzone Salvatore Cuzzocrea \\ Emanuela Esposito
}

Department of Chemical, Biological, Pharmaceutical and Environmental Sciences, University of Messina, Messina, Italy

\author{
Keywords \\ Xyloglucan · Pea protein · Skin barrier · Permeability · \\ Staphylococcus aureus
}

\begin{abstract}
Objective: The skin acts as a mechanical and protective barrier against viral, fungal, and bacterial infections. Skin conditions such as atopic dermatitis and psoriasis are characterized by alterations of the skin barrier, often caused by injury and by bacterial infections. In the last years, non-pharmacological interventions have gained great importance in epidermis-related diseases. Xyloglucan (XG) is a polysaccharide that possesses a "mucin-like" molecular structure that confers mucoadhesive properties, allowing XG-containing formulations to act as a protective barrier for the management of different diseases. Moreover, there is also increasing interest in the use of proteins due to their film-forming features. This study aimed to evaluate the barrier-protective properties of a product containing XG and pea protein (PP) in an in vitro model, assessing its effects on the membrane permeability of keratinocytes infected by Staphylococcus aureus. Methods: $\mathrm{HaCaT}$ keratinocytes were pretreated with XG and PP for $3 \mathrm{~h}$ and then infected with S. aureus cells $\left(10^{6}\right.$ bacteria/ well) at a multiplicity of infection of 10 for $1 \mathrm{~h}$. The number
\end{abstract}

of bacterial colonies and membrane integrity were measured, respectively. Results: We observed that pretreatment with XG and PP in human $\mathrm{HaCaT}$ keratinocytes infected with S. aureus significantly increased trans-epithelial electrical resistance (a marker of skin barrier function) measurement, reduced lucifer yellow (a marker of membrane integrity) permeation across the monolayer, and released lactate dehydrogenase (a marker of tissue damage). Moreover, XG and PP pretreatment was able to reduce bacterial adherence, avoiding S. aureus infection. Conclusion: In summary, we demonstrated that the product containing XG and PP was able to maintain barrier permeability preserving its integrity, and therefore, it can be considered as an interesting approach for the management of epidermis-related diseases.

(c) 2020 S. Karger AG, Basel

\section{Introduction}

The skin is constantly exposed to a variety of external noxious agents. One of the most important functions of the epidermis is to generate a permeable barrier against viral, fungal, and bacterial infections and prevent excess trans-epidermal water loss $[1,2]$. The loss of these functions leads to the colonization of different bacteria, in- karger@karger.com

(C) 2020 S. Karger AG, Base

www.karger.com/spp

Karger ${ }^{\prime}=$
Emanuela Esposito

Department of Chemical, Biological, Pharmaceutical and Environmental Sciences University of Messina, Viale Ferdinando Stagno d'Alcontres 31 IT-98166 Messina (Italy)

eesposito@unime.it 
cluding Staphylococcus aureus $[3,4]$. S. aureus uses adhesion molecules to adhere to the skin producing extracellular vesicles containing immunogenic compounds and serine proteases that can further damage the skin barrier, all of which alter the epidermal permeability [5]. Therefore, appropriately formulated skin products are needed to maintain skin barrier integrity, preventing acute or chronic inflammatory and infectious skin diseases. Xyloglucan (XG) belongs to a new class of products defined as "mucosal protectors," which form a protective film and exercise a mechanical effect [6]. XG is a hemicellulose of plant origin, extracted from seeds of the tamarind tree (Tamarindus indica). It has been shown that XG is able to restore physiological functions of the intestinal wall [7]; $\mathrm{XG}$ forms a protective film which facilitates the mucosal resistance to pathological invaders, restoring normal function. This barrier effect decreases the permeability of the intestinal mucosa and avoids the adherence and proliferation of pathogens [7]. Furthermore, in vitro studies have shown that XG increases trans-epithelial electrical resistance (TEER) following Escherichia coli infection [7]. The TEER is an index that assesses good functionality of the mucosal tight junctions and therefore confirms the ability of XG to neutralize mucosal permeability after $E$. coli infection [8]. Moreover, considerable importance was given to the proteins extracted from the Pisum sativum plant. Pea protein (PP) is a fiber-rich legume that is low in fat and boasts an extraordinary aminoacidic profile conferring to PP favorable health properties due to its film-forming features $[6,9]$. Given the beneficial protective properties of XG and PP towards mucosal barriers, the aim of this study was to evaluate the barrier-protective properties of a product containing XG and PP in an in vitro model, testing the permeability of keratinocyte monolayers upon S. aureus infection.

\section{Materials and Methods}

\section{Materials}

All chemicals were obtained from the highest grade of commercial sources. The product containing XG and PP was kindly provided by DEVINTEC SAGL (Lugano, Switzerland). S. aureus (ATCC 29213) was purchased by ATCC materials resource. Human immortalized keratinocytes (HaCaT cell line) (CLS Cell Lines Service, 300493) were used for the study. For Franz diffusion cell studies, we used XG+PP at the dose of $200 \mathrm{mg}$, dissolved in a physiological buffer.

\section{S. aureus Culture}

For the experimental infection, $S$. aureus strain was grown to the exponential phase (about $1 \times 10^{9} \mathrm{CFU} / \mathrm{mL}$ ) in brain heart infu- sion broth at $37^{\circ} \mathrm{C}$ overnight with shaking, and harvested by centrifugation $(5,000 \mathrm{~g}$ for $5 \mathrm{~min})$, washed ( $3 \times$ in PBS), and suspended to the required number in fresh, serum- and antibiotic-free RPMI 1640 medium. The viable count for infection was made through a spread plate technique.

\section{S. aureus Infection}

HaCaT keratinocytes were grown in RPMI 1640 medium supplied with $10 \% \mathrm{FBS}$ at $37^{\circ} \mathrm{C}$ in $5 \% \mathrm{CO}_{2}$. The keratinocytes monolayers were grown in 12-well tissue culture plates and infected with SA cells $\left(10^{6}\right.$ bacteria/well $)$ at a multiplicity of infection of 10 for $1 \mathrm{~h}$ at $37^{\circ} \mathrm{C}$ [10]. Extracellular bacteria were removed by washing the keratinocytes 3 times with PBS, the medium was replaced with $1 \%$ FBS-RPMI 1640 medium containing $50 \mu \mathrm{g} / \mathrm{mL}$ of gentamicin to kill the extracellular SA bacteria for $1 \mathrm{~h}$. Subsequently, the cells were washed 3 times with PBS to remove the gentamicin. XG+PP at chosen concentrations was added to cells $3 \mathrm{~h}$ before SA infection $\left(1 \mathrm{~h}\right.$ at $\left.37^{\circ} \mathrm{C}\right)$. After that, infected cells were washed 3 times with PBS and then incubated for $15 \mathrm{~min}$ at $37^{\circ} \mathrm{C}$ with $0.02 \%$ Triton X-100 to lyse the keratinocytes and release the intracellular bacteria into the lysate. Various dilutions of the lysates were plated on brain heart infusion agar plates and incubated overnight at $37^{\circ} \mathrm{C}$. The colonies were counted to determine the rate of infection. To analyze the number of surviving bacteria compared to the initial number of intracellular bacteria (time zero), the relative number of CFU was calculated as follows: number of CFU at $4 \mathrm{~h} /$ number of CFU at time zero.

\section{Experimental Groups}

Group 1: control + vehicle: PBS was added to $\mathrm{HaCaT}(n=12)$

Group 2: control + XG+PP: XG+PP was added to $\mathrm{HaCaT}(n=12)$

Group 3: S. aureus: keratinocyte monolayers were infected with $S$. aureus cells $\left(10^{6}\right.$ bacteria/well $)$ at a multiplicity of infection of 10 for $1 \mathrm{~h}$ at $37^{\circ} \mathrm{C}(n=12)$

Group 4: $S$. aureus + XG+PP: XG+PP was added to cells $3 \mathrm{~h}$ before SA infection $(n=12)$

\section{LDH Assay}

The membrane integrity of the keratinocytes was determined by measuring the lactate dehydrogenase (LDH) activity present in the culture supernatant following $S$. aureus infection. The assay was performed using a CytoTox 96 nonradioactive cytotoxicity assay kit (Promega, Madison, WI, USA), according to the manufacturer's instructions. LDH was measured at $492 \mathrm{~nm}$ absorbance using a standard 96-well plate reader. LDH release was expressed as units per liter of supernatant. The percentage of cytotoxicity was calculated as follows: ([experimental release - spontaneous release]/[total release - spontaneous release] $) \times 100$, where a spontaneous release is the amount of $\mathrm{LDH}$ activity in supernatants of cells incubated in medium alone, and total release is the $\mathrm{LDH}$ activity measured in cell lysates [11].

\section{Lucifer Yellow Permeation}

Lucifer yellow (LY) was used as a membrane integrity marker. Cells were plated onto 96-transwell plates with polycarbonate membrane inserts at a seeding density of $50,000 \mathrm{cells} / \mathrm{cm}^{2}$. The cells were grown to confluence and treated on the 5 th day with dilutions of XG+PP for $4 \mathrm{~h}$, then were infected with SA for $1 \mathrm{~h}$. The monolayer integrity was evaluated by measuring the LY permeation across the barrier, as previously described [12]. The LY con- 


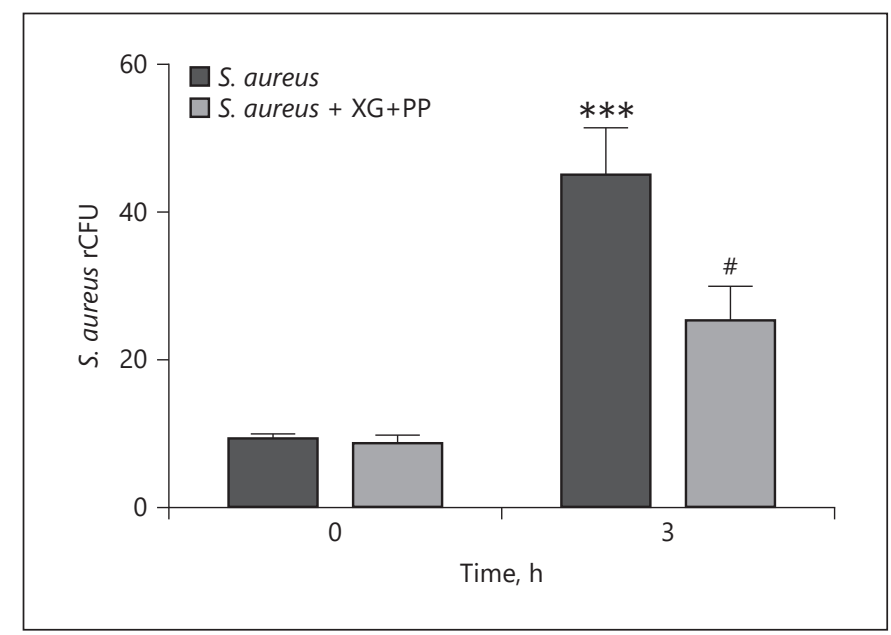

Fig. 1. Evaluation of intracellular bacteria. The relative number of CFU (rCFU) was estimated by counting the number of CFU at $\mathrm{T}_{0}$ and $\mathrm{T}_{4 \mathrm{~h}}$. The association $\mathrm{XG}+\mathrm{PP}$ reduced the number of $\mathrm{rCFU}$ compared to control group. Data are representative of at least three independent experiments; ${ }^{* * *} p<0.001$ versus control; ${ }^{*} p<0.05$ versus $\mathrm{XG}+\mathrm{PP}$

centrations in the apical and basolateral divisions were determined by measuring their fluorescence intensities compared to the standard curves of LY, at excitation and emission wavelengths of 485 and $529 \mathrm{~nm}$, respectively, using a microplate reader.

\section{TEER Measurement}

A quantitative measure describing the barrier integrity and the electrical, ohmic resistance of the cell layer was performed. The traditional method for measuring TEER values is to use STX2 electrodes using a 4-point measurement system on Transwell permeable inserts [13]. One set of electrodes was placed in the basolateral compartment and the other was placed in the apical compartment [14]. For measurement of TEER, the cells were seeded at a high plating density $\left(1.25 \times 10^{5}\right.$ cells $\left./ \mathrm{cm}^{2}\right)$ on clear polyester Transwell permeable supports (Corning Glass) in growth medium. Medium (apical and basal) was changed every $24 \mathrm{~h}$ before and during treatments. The association $\mathrm{XG}+\mathrm{PP}$ was added to the culture medium when TEER reached a plateau (2-3 weeks after seeding, $\sim 3,000 \mathrm{ohms} \times \mathrm{cm}^{2}$ ). An EVOM epithelial volt/ohmmeter with STX (chopstick) electrodes (World Precision Instruments, Sarasota, FL, USA) was used to measure TEER.

\section{Franz Diffusion Cell Studies on Hairless Mouse Skin Infected}

by S. aureus

The association $\mathrm{XG}+\mathrm{PP}$ barrier effect on hairless mouse skin infected by $S$. aureus was observed using the Franz diffusion cell method [15]. Mouse skin was set up in Franz cells apparatus. Briefly, frozen $\left(-20^{\circ} \mathrm{C}\right)$ skin was defrosted, the underlying fat was removed, then the epidermis was cleaned with distilled water and dried. The chamber receptor cell was filled with $1 \mathrm{~mL}$ of $\mathrm{PBS}$ and allowed to equilibrate at $32 \pm 2{ }^{\circ} \mathrm{C}$ in the heated magnetic block for $30 \mathrm{~min}$. The skin was cut into $3.5-\mathrm{cm}$ diameter pieces sufficient to cover the area of the receptor and was fixed between the donor and the receptor chamber [16]. The associa-

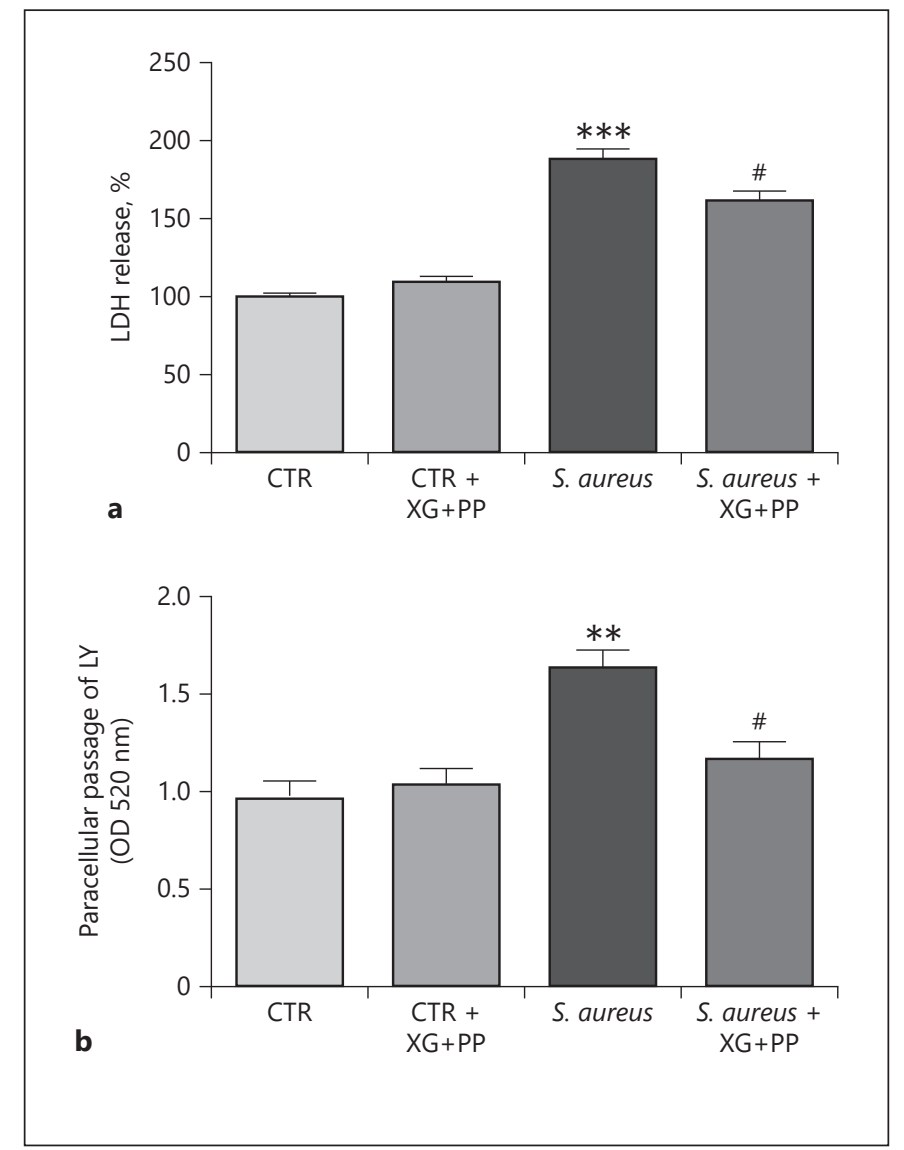

Fig. 2. a, b Evaluation of XG+PP on LDH and LY tests. The pretreatment with $\mathrm{XG}+\mathrm{PP}$ reduced the percentage of $\mathrm{LDH}$ released in HaCaT keratinocytes (a). The luciferase measurement highlighted a reduction of LY permeation after the pretreatment with dilutions of $\mathrm{XG}+\mathrm{PP}$ compared to $\mathrm{HaCaT}$ infection with S. aureus (b). Data are representative of at least three independent experiments. ${ }^{* * *} p<0.001$ vs. control (CTR); ${ }^{* *} p<0.01$ versus CTR; ${ }^{*} p<0.05$ versus $\mathrm{XG}+\mathrm{PP}$.

tion $\mathrm{XG}+\mathrm{PP}$ was placed on the skin surface in the donor compartment and was brushed to cover the whole surface uniformly. $S$. aureus was harvested and suspended in PBS to determine the bacterial density $\left(1.5 \times 10^{7} \mathrm{CFU} / 100 \mu \mathrm{L}\right)$ and then was added to the upper well of the skin. After $30 \mathrm{~min}$, the skin surface was washed twice with $6 \mathrm{~mL}$ of PBS to recover $S$. aureus and then serial dilutions of the washings were added to BD Mannitol Salt Agar plates followed by CFU counting to evaluate $S$. aureus proliferation [17]. After $24 \mathrm{~h}$, the formation of colonies was observed. A control experiment was performed by adding on the skin surface only S. aureus; CFUs were counted after washing as explained above.

\section{Statistical Analysis}

All values are expressed as mean \pm SEM. The results were analyzed by one-way ANOVA followed by a Bonferroni post hoc test for multiple comparisons. A value of $p \leq 0.05$ was predetermined as the criterion of significance. 


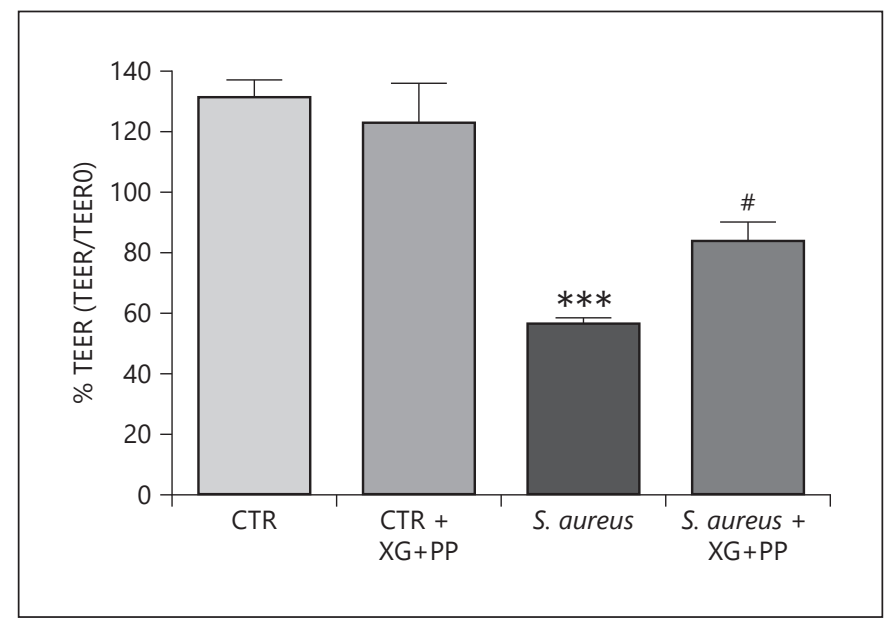

Fig. 3. Evaluation of $X G+P P$ on TEER measurement. The pretreatment with XG+PP prevented a decrease in TEER values compared to HaCaT infection with $S$. aureus. Data are representative of at least three independent experiments. ${ }^{* * *} p<0.001$ versus control (CTR); ${ }^{\#} p<0.05$ versus $\mathrm{XG}+\mathrm{PP}$.

\section{Results}

Evaluation of S. aureus CFU on Infected Keratinocytes

The relative number of CFU was estimated by plating out the lysate of infected keratinocytes and counting the number of CFU at each time point $\left(\mathrm{T}_{0}\right.$ and $\left.\mathrm{T}_{4 \mathrm{~h}}\right)$. The relative number of CFU is the difference between the initial number of intracellular bacteria and the number at $4 \mathrm{~h}$. As shown by Figure 1, XG+PP was able to avoid S. aureus infection by reducing bacteria adherence (Fig. 1).

\section{Evaluation of Keratinocyte Membrane Integrity with \\ LDH Assay and LY Permeation Test}

The release of $\mathrm{LDH}$ was performed to investigate keratinocyte membrane integrity. $\mathrm{LDH}$ release significantly increased in the cell culture supernatant after incubation with $S$. aureus compared with the control group. The addition of $\mathrm{XG}+\mathrm{PP}$ was able to counteract the damaging effects of $S$. aureus infection reducing the percentage of LDH released in $\mathrm{HaCaT}$ keratinocytes (Fig. 2a). A LY permeation study was performed to test the effects of XG+PP on $\mathrm{HaCaT}$ cell monolayer intactness. S. aureus infection, after $24 \mathrm{~h}$, significantly increased the paracellular flux of $\mathrm{LY}$, while application of XG+PP reduced LY permeation across the monolayer (Fig. $2 \mathrm{~b}$ ).

\section{Evaluation of Skin Barrier Function with TEER Test}

$\mathrm{HaCaT}$ infection with $S$. aureus significantly reduced TEER measurement, while the administration of XG+PP

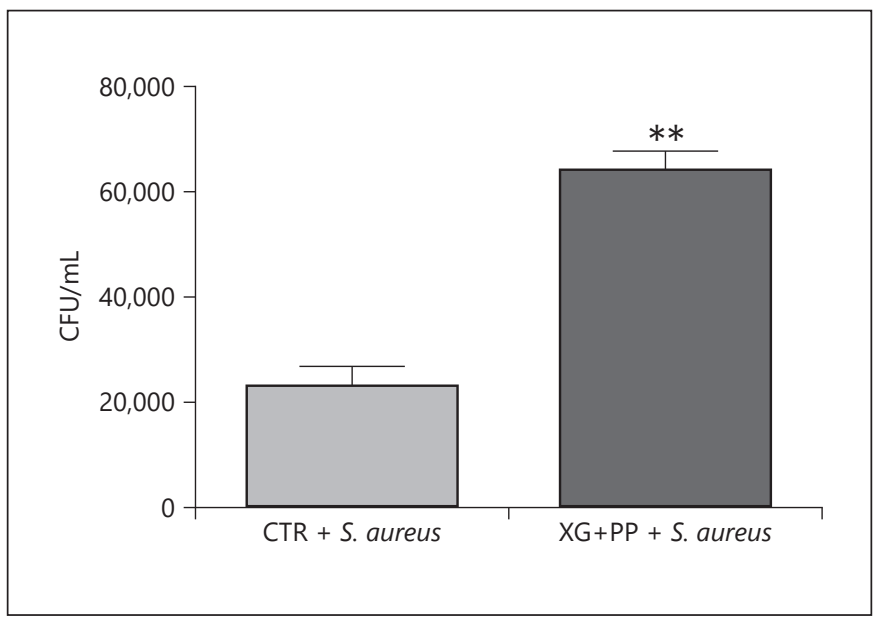

Fig. 4. Evaluation of $\mathrm{XG}+\mathrm{PP}$ barrier effect on mouse skin with Franz cell method. The pretreatment with $\mathrm{XG}+\mathrm{PP}$ prevented the attachment and the penetration of $S$. aureus compared to control group (CTR). Data are representative of at least three independent experiments. ${ }^{* *} p<0.01$ versus CTR.

prevented TEER decrease, enabling the maintenance of skin barrier function and integrity (Fig. 3).

\section{Evaluation of Barrier Effect on Mouse Skin Infected by}

S. aureus Using Franz Diffusion Cell

The XG+PP barrier effect on hairless mouse skin infected by $S$. aureus was observed using the Franz diffusion cell method. The bacterial burden was evaluated by plating serial dilutions of S. aureus onto the BD Mannitol Salt Agar dish counting the CFU to evaluate bacterial proliferation. It was observed that $\mathrm{XG}+\mathrm{PP}$ decreased the attachment and penetration of $S$. aureus onto the surface of the skin, as demonstrated by the increased number of CFU counted, as shown in Figure 4.

\section{Discussion and Conclusions}

The disruption of the skin barrier function was recently associated with a variety of diseases, such as atopic dermatitis, contributing to a greater risk of contracting bacterial infections, in particular by $S$. aureus $[18,19]$. Therefore, compounds able to preserve barrier integrity could represent a promising approach to counteract skin pathologies. The aim of this study was to demonstrate the barrier-protective properties of a product containing XG and PP through an in vitro permeability model of keratinocyte membrane infected by S. aureus. Our data demonstrated that the number of internalized bacterial colo- 
nies drastically increased within just $3 \mathrm{~h}$ in infected keratinocytes. On the contrary, the pretreatment with XG+PP reduced bacterial adherence to keratinocyte membranes, thereby avoiding $S$. aureus infection. Membrane integrity plays an important role in regulating epithelial proliferation and differentiation by modulating dermal-epidermal interaction. Moreover, it is a fundamental requirement that ensures structural support to the skin tissue and also offers functional inputs that modulate cellular function [20]. Therefore, in this study we evaluated $\mathrm{LDH}$ and luciferase levels to highlight the loss of membrane integrity in skin disorders caused by staphylococcal infection. The presence of LDH in the extracellular fluid is an indicator of cell death and loss of membrane integrity. The release of $\mathrm{LDH}$ that was found in the supernatants was proportional to cellular damage [21]. However, XG+PP significantly reduced levels of released $\mathrm{LDH}$ in keratinocytes infected by S. aureus. LY is a particularly useful marker to study the trans-epithelial transport and membrane integrity [22]. Surprisingly, the addition of XG+PP has shown to reduce LY permeation across the monolayer of keratinocytes infected by $S$. aureus. Therefore, thanks to the protective properties, XG and PP demonstrated the ability, through a mechanical effect, to restore the integrity of the membrane, avoiding skin disorders that can be induced by bacteria. Skin permeability is a fundamental requirement to guarantee the barrier function. In fact, many aspects related to the conditions of the stratum corneum can alter the skin permeability, as well as the integrity and presence of skin diseases [23]. Pathological processes can compromise the skin barrier in more ways: influencing directly the composition of proteins and lipids, for example in atopic dermatitis [24], or causing a modification of the stratum corneum by altering the proliferation process of the corneocytes seen in psoriasis [25]. The loss of this equilibrium leads to an impaired function of the skin barrier, leading to an activation of the immune response and induction of inflammation [26]. This study demonstrated that the permeability of keratinocyte monolayers was markedly compromised by $S$. aureus infection. The addition of XG+PP has shown to preserve TEER. This test clearly reflects the degree of cell confluency including the formation of cellular junctions [27] and provides precise information on barrier characteristics before and after exposure to the staphylococcal infection. Therefore, these results highlight the preserved functionality of mucosal tight junctions and confirm the ability of $\mathrm{XG}+\mathrm{PP}$ to maintain a permeable skin barrier function after skin damage. XG and PP possess mucoadhesive and mucomi- metic properties to form a bio-protective film that avoids pathological aggression and helps to restore the normal function of the skin barrier $[7,28]$. To confirm the nonpharmacological mechanism of action, this study showed that the application of XG+PP significantly decreased the attachment and penetration of $S$. aureus on the surface of the mouse skin.

In conclusion, these results demonstrated that the product containing $\mathrm{XG}+\mathrm{PP}$ has significant effects on the maintenance of the integrity and permeability of the keratinocyte layers. This study clearly demonstrated that XG and PP, thanks to their protective properties, can be used for the management of skin disorders by preserving the physiological function of the skin barrier.

\section{Statement of Ethics}

Skin from male CD1 mice (Envigo, Milan, Italy) weighing 20$25 \mathrm{~g}$ were used for Franz cell study. This study was approved by the University of Messina Review Board for the care of animals (Protocol number $8 / \mathrm{U}$-apr16). Animal care was in conformity with current legislation for the protection of animals used for scientific purposes (Directive 2010/63/EU, April 9, 2016) and the ARRIVE guidelines.

\section{Conflict of Interest Statement}

The study was financed by Devintec SAGL, Switzerland. The authors have no other relevant affiliations or financial involvement with any organization or entity with a financial interest, or financial conflict with the subject matter or materials discussed in the manuscript apart from those disclosed. No writing assistance was utilized in the production of the manuscript.

\section{Author Contributions}

E.E. and S.C. planned the experiments. M.L., A.F., G.C., S.A.S., and A.A. performed experiments. M.C. and I.P. analyzed the results and prepared the manuscript. All authors read and approved the final manuscript.

\section{Data Availability Statement}

The authors declare that all data and materials supporting the findings of this study are available within the article. The data that support the findings of this study are available from the corresponding author upon reasonable request. 


\section{References}

1 Aszterbaum M, Menon GK, Feingold KR, Williams ML. Ontogeny of the epidermal barrier to water loss in the rat: correlation of function with stratum corneum structure and lipid content. Pediatr Res. 1992 Apr;31(4 Pt 1):308-17.

2 Aberg KM, Man MQ, Gallo RL, Ganz T, Crumrine D, Brown BE, et al. Co-regulation and interdependence of the mammalian epidermal permeability and antimicrobial barriers. J Invest Dermatol. 2008 Apr;128(4):917-25.

3 Palmer CN, Irvine AD, Terron-Kwiatkowski A, Zhao Y, Liao H, Lee SP, et al. Common loss-of-function variants of the epidermal barrier protein filaggrin are a major predisposing factor for atopic dermatitis. Nat Genet. 2006 Apr;38(4):441-6.

4 Menon GK, Feingold KR, Elias PM. Lamellar body secretory response to barrier disruption. J Invest Dermatol. 1992 Mar;98(3):279-89.

5 Zettersten E, Man MQ, Sato J, Denda M, Farrell A, Ghadially R, et al. Recessive x-linked ichthyosis: role of cholesterol-sulfate accumulation in the barrier abnormality. J Invest Dermatol. 1998 Nov;111(5):784-90.

6 Piqué N, Gómez-Guillén MD, Montero MP. Xyloglucan, a Plant Polymer with Barrier Protective Properties over the Mucous Membranes: an Overview. Int J Mol Sci. 2018 Feb; 19(3):E673.

7 Gnessi L, Bacarea V, Marusteri M, Piqué N. Xyloglucan for the treatment of acute diarrhea: results of a randomized, controlled, open-label, parallel group, multicentre, national clinical trial. BMC Gastroenterol. 2015 Oct;15(1):153.

8 Lodemann U, Strahlendorf J, Schierack P, Klingspor S, Aschenbach JR, Martens H. Effects of the Probiotic Enterococcus faecium and Pathogenic Escherichia coli Strains in a Pig and Human Epithelial Intestinal Cell Model. Scientifica (Cairo). 2015;2015:235184.
9 Mathai JK, Liu Y, Stein HH. Values for digestible indispensable amino acid scores (DIAAS) for some dairy and plant proteins may better describe protein quality than values calculated using the concept for protein digestibilitycorrected amino acid scores (PDCAAS). Br J Nutr. 2017 Feb;117(4):490-9.

10 Wiegand C, Abel M, Ruth P, Hipler UC. $\mathrm{HaCaT}$ keratinocytes in co-culture with Staphylococcus aureus can be protected from bacterial damage by polihexanide. Wound Repair Regen. 2009 Sep-Oct;17(5):730-8.

11 Ingrassia I, Leplingard A, Darfeuille-Michaud A. Lactobacillus casei DN-114 001 inhibits the ability of adherent-invasive Escherichia coli isolated from Crohn's disease patients to adhere to and to invade intestinal epithelial cells. Appl Environ Microbiol. 2005 Jun;71(6):2880-7.

12 Duong QV, Kintzing ML, Kintzing WE, Abdallah IM, Brannen AD, Kaddoumi A. Plasma Rich in Growth Factors (PRGF) Disrupt the Blood-Brain Barrier Integrity and Elevate Amyloid Pathology in the Brains of 5XFAD Mice. Int J Mol Sci. 2019 Mar;20(6):E1489.

13 Benson K, Cramer S, Galla HJ. Impedancebased cell monitoring: barrier properties and beyond. Fluids Barriers CNS. 2013 Jan;10(1):5.

14 Gao Y, Li S, Wang J, Luo C, Zhao S, Zheng N. Modulation of Intestinal Epithelial Permeability in Differentiated Caco-2 Cells Exposed to Aflatoxin M1 and Ochratoxin A Individually or Collectively. Toxins (Basel). 2017 Dec; 10(1):E13.

15 Seo JE, Kim S, Kim BH. In vitro skin absorption tests of three types of parabens using a Franz diffusion cell. J Expo Sci Environ Epidemiol. 2017 May;27(3):320-5.

16 Ingram RJ, Bartlett A, Brown MB, Marriott C, Whiffield RJ. Dimethicone barrier cream prevents infection of human skin by schistosome cercariae: evidence from Franz cell studies. J Parasitol. 2002 Apr;88(2):399-402.

17 Ng SF, Rouse J, Sanderson D, Eccleston G. A Comparative Study of Transmembrane Diffusion and Permeation of Ibuprofen across Synthetic Membranes Using Franz Diffusion Cells. Pharmaceutics. 2010 May;2(2):209-23.
18 Cho SH, Strickland I, Boguniewicz M, Leung DY. Fibronectin and fibrinogen contribute to the enhanced binding of Staphylococcus aureus to atopic skin. J Allergy Clin Immunol. 2001 Aug; 108(2):269-74.

19 Rerknimitr P, Otsuka A, Nakashima C, Kabashima $\mathrm{K}$. The etiopathogenesis of atopic dermatitis: barrier disruption, immunological derangement, and pruritus. Inflamm Regen. 2017 Jun;37(1):14.

20 Iriyama S, Matsuura-Hachiya Y, Tsunenaga M. Influence of epidermal basement membrane integrity on cutaneous permeability barrier function. J Dermatol Sci. 2018 Jul; 91(1):110-2.

21 Rodríguez A, Webster P, Ortego J, Andrews NW. Lysosomes behave as Ca2+-regulated exocytic vesicles in fibroblasts and epithelial cells. J Cell Biol. 1997 Apr;137(1):93-104.

22 Hanani M. Lucifer yellow - an angel rather than the devil. J Cell Mol Med. 2012 Jan;16(1): 22-31.

23 Williams AC, Barry BW. Penetration enhancers. Adv Drug Deliv Rev. 2004 Mar;56(5): 603-18.

24 Williams ML. Ichthyosis: mechanisms of disease. Pediatr Dermatol. 1992 Dec;9(4):365-8.

25 Lowes MA, Suárez-Fariñas M, Krueger JG. Immunology of psoriasis. Annu Rev Immunol. 2014;32(1):227-55.

26 Lopetuso LR, Scaldaferri F, Bruno G, Petito V, Franceschi F, Gasbarrini A. The therapeutic management of gut barrier leaking: the emerging role for mucosal barrier protectors. Eur Rev Med Pharmacol Sci. 2015;19(6): 1068-76.

27 Casettari L, Vllasaliu D, Mantovani G, Howdle SM, Stolnik S, Illum L. Effect of PEGylation on the toxicity and permeability enhancement of chitosan. Biomacromolecules. 2010 Nov;11(11):2854-65.

28 Kulkarni AD, Joshi AA, Patil CL, Amale PD, Patel HM, Surana SI, et al. Xyloglucan: A functional biomacromolecule for drug delivery applications. Int J Biol Macromol. 2017 Nov;104(Pt A):799-812. 